\title{
QoS Analysis in Mobile Ad Hoc Networks Using Queueing Theoretic Approaches: A Review
}

\author{
Aleem Ali \\ School of ICT, GBU, \\ Greater Noida, U.P, India \\ Neeta Singh \\ School of ICT, GBU, \\ Greater Noida, U.P, India
}

\begin{abstract}
A Mobile Adhoc NETwork (MANET) is a collection of wireless communication nodes that wish to communicate with each other without the aid of any fixed infrastructure and predetermined topology of wireless links. To support multimedia or real time applications, it is highly desirable that a MANETs must have provision of Quality of Service (QoS). However, the provision of QoS in MANETs is a big challenging task. Queueing network models have been successfully used for performance modeling of MANETs. Various performance measures such as end to end delay, throughput, jitter, packet drop rate etc. were used to study and analyze in MANETs. This paper presents a review of ongoing research related to the provision of QoS in MANETs using queueing theoretic approaches.
\end{abstract}

Keywords: Queueing network models, QoS, MANETs, end-to-end delay, packet drop fraction (PDF)

\section{INTRODUCTION}

A MANET is a mobile mesh network, it is an auto- configurable and self organize network of mobile devices, inter-connected through wireless links and communicate each other using single hop as well as multi-hop path. MANET is a refined version of traditional wireless network and considered as a dominating network in the upcoming future. Figure 1 shows the general architecture of MANET.

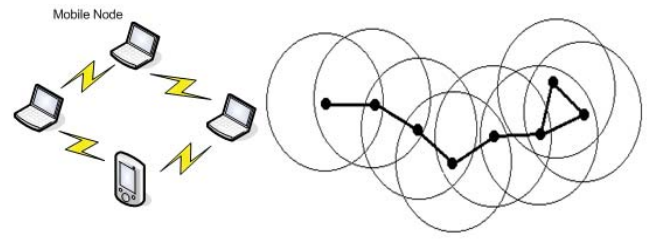

Figure 1: A general view of MANET with Multi-hop facility

With the advent of wireless prevalence, there are lot of applications coming and adapting to wireless techniques to communicate and getting dominance over wired network.. In recent times, with the proliferation of peer to peer, infrastructure-less and inexpensive nature of MANETs, researchers attention have been shifted upto a far extent to provide assurance related to QoS in the networks, since MANETs are very much capable of supporting real time traffic as well as multimedia application [1][2]. However, modelling of an ad hoc network with mobile nodes is a challenging task, due to the modeling of the behavior of wireless links as well as behavior of nodes. The performance of QoS based solutions is hugely influenced by several design issues. For an application to be QoS enabled, a route with ample resources to fulfill rigid QoS demands should be used [3].

To accommodate such applications, some specific QoS parameters like delay, bandwidth, energy etc. needs to be taken into account which can utilize the limited resources efficiently. None of the traditional approach or routing protocol deals with these characteristics efficiently in their implementation. With the use of various queueing theoretic approaches, performance modeling on various parameters metrics could be used to analyze the QoS requirements in various dynamic network scenarios.

Baskar et. al. [4] proposes Enhanced Probabilistic Adhoc on Demand Distance Vector (EPAODV) routing protocol, which determines the performance of the network and also capable of solving broadcast storm problem 
of the Adhoc on Demand Distance Vector (AODV). The proposed algorithm which is based on a queueing model $\mathrm{M} / \mathrm{M} / \mathrm{C}: \infty / \mathrm{FIFO}$, enhances better results such as decreasing the end to end delay, increasing throughput and then enhancing data delivery ratio compare to the existing protocols. Further the network performance like packet delay is minimized and data delivery ratio is maximized, if the network is kept high.

Seema et. al. [5] proposes a G/G/1 queuing network model to gain insights for closed form expression on the average end to end delay in a multi hop wireless ad hoc networks. Enass et. al [6] showed that each node of the MANET was modeled as a queuing system to serve the packets that reaching according to certain arrival rate. Many performance metrics such as average end-to-end delay, throughput, packets loss and packet delivery fraction were used as comparison indications for two queueing mechanisms (DropTail, REM). The network PDF in REM is better than with the DropTail. Vishal et. al [7] proposed a novel approach of modelling mobile ad hoc networks using open queueing network model DBMAP/D/1/N. Nodes of a queueing network are static and hence they do not move.

Khandelwal et. al [8] proposes a probabilistic routing protocol (PAODV) that uses M/M/C:œ/FIFO queueing model with infinite packet arrivals at each node. The results have been taken for end to delay, throughput and then data delivery ratio which showed better PAODV efficiency over AODV.

Ahyoung et. al [9] suggested a M/M/1/B queueing network model for a multimedia communication based on adaptive gossip algorithm which analyzes the queueing delay with respect to transmission range, number of nodes, behavior of routing and MAC protocols. Adaptive gossip algorithm with comparative to flooding algorithm, for small low traffic network has significantly, $70 \%$ less delay and for large high traffic network having large packet size such as a video traffic has, $56 \%$ less delay. Hence, this algorithm is adequately satisfy QoS requirements in video/voice communications as compare to flooding.

Bisnik N. et al [10] studied a open G/G/1 queueing network model with infinite buffer to estimate the average end to end in a multi hop wireless ad hoc network using diffusion approximation method. However, in a real time system each node of wireless ad hoc network has limited buffer. Ell Khoury R. et al [11] proposed a cross layer scheme using G/G/1 queueing model in wireless multi hop ad hoc network for decreasing the end to end delay of real time streaming media and packets as well that arrive after their schedule timing. However, their approach was focused on theoretical work.

Kamal K. Sharma et. al [12] focuses on studying M/D/1 queue for In-vehicle multimedia network that approximates the maximum achievable throughput and end to end delay at each node in order to improve QoS. Ozdemir M. et. al [13] studied M/MMG1/1/K queuing model for traffic used in order to compute the expected end-to-end packet delay over single hop communication network. Dey A.k et. al [14], have shown average end to end delay-throughput tradeoffs in wireless ad hoc networks with mobile and stationary nodes and resulted, the average delay and throughput are related by $\mathrm{D}(\mathrm{n})=\Phi(\mathrm{nT}(\mathrm{n}))$, for a wireless network with stationary nodes where $D(n)$ and $T(n)$ are the average delay and throughput respectively. Many more ongoing studies have proposed queuing theoretic models for performance analysis of the IEEE 802.11 MAC. J. Li et. al [15] modeled the service time of a node as a Markov general arrival process in IEEE 802.11 MAC based ad hoc network.

However, so far many queueing theoretic models have been applied for current service discovery on MANETs but still QoS is a core issue. Wen Y. et. al [16] proposes a M/M+/1 model for SCN4M discovery system which achieve optimal QoS at any kind of environmental changes. Barbeau M. et. al [17], defines service discovery strategies as time-dependent Post- Query protocols that implemented in rounds and can be applied to analyze network costs in communication time. Chakraborty D. et. al [18] applies M/G/c/c queuing theoretic model to the service discovery protocols in ad hoc networks which predict the behaviour of service cache at node level. The correctness of the queueing model is verified by experiments that approximates the cache usage of service cache in a mobile device participating in ad hoc service discovery.

The rest of the paper is organised as follows: In the next section, we have discussed challenges in QoS provisioning over MANETs. Section 3 devoted to various issues in MANETs. In section 4, we have provided a detail about queueing approach to achieve QoS in terms of different parameters related to QoS in MANETs. The last section finally conclude the paper.

\section{ISSUES OF MANETs}

This section presents numerous issues encountered while transferring real time traffic over MANETs. Quality of Service (QoS): QoS is one of the major issue in MANETs because of its dynamic nature of mobile nodes (MNs), frequent changes in topology, energy constraints and bandwidth limitations. Usually, QoS is pre specified service performance constraints to user in terms of end-to-end delay, delay variance (jitter), available 
bandwidth, and probability of packet loss etc. These constraints require the offering of guaranteed service quality [19].

Routing: In MANETs, each mobile node comprises as a router and forwards packets to enable information giving out between nodes [1]. However, finding an efficient route while adhering to multiple QoS requirement is typically difficult and in many cases is considered as a NP-complete problem [20].

Security issue: MANET are more prone to suffer security which may cause routing loops, message distortion, advertisements of fake or old routing table updates. Security may have many unclear issues that are important to solve to make the network into a good solution.

Addressing scheme: The scheme which is being used in mobile IP doesn't apply to MANET due to their decentralized nature. The network topology keeps changing dynamically and hence the addressing scheme used is quite significant. A dynamic network topology entails a ubiquitous addressing scheme, which avoids any duplicate addresses. Mobile IP is currently being used in cellular networks where a base station handles all the node addressing [21].

\section{CHALLENGES IN QOS PROVISIONING OVER MANETs}

This section provides a brief description of major challenges encountered in MANETs while providing QoS enhancements:

Resource constraints: The mobile devices that constitute the entire MANET system have bandwidth constraint, limited available battery power, processing ability, speed of operation and limited on-board memory space. Also, compared to wireline networks, the channel capacity is lower in wireless networks. Due to above mentioned constraints; resources need to be managed efficiently for their best utilization in traditional as well as QoS aware scenario.

Uncertain physical features: Wireless channels are quite prone to errors that occur due to interference because of simultaneous transmission by neighbour nodes, fading, noise etc.

Decentralized network architecture: Inherent nature of MANETs i.e. being easy to deploy, quite inexpensive and infrastructure-less seems extremely promising for being used in variety of domains. Being decentralized, MANET hosts are required to transmit their QoS state information to the remaining hosts, thereby making QoS aware protocols quite complex and prone to overheads.

Contention for channels: In MANETs, nodes talk to each other through shared channels. This communication is necessary so that most updated information about network states, routes and nodes must be exchanged among nodes. This frequent communication will eventually result in high collision due to interference among node signals causing increase in battery consumption and delay along with decrease in packet delivery ratio and link bandwidth utilization.

Multi-hop mode of communication: Apart from being the host devices, MANET nodes also function as routers and perform the basic operations of forwarding packets between source and destination. Prior to forwarding, an optimal route is discovered with the help of intermediate nodes. The data is then transmitted on the discovered path hop by hop through intermediate nodes; hence if failure occurs in even a single node, the entire MANET transmission can fail.

Imprecise state information: MANET maintain both link-specific as well as flow-specific state information. The link-specific state information includes delay, delay jitter, bandwidth, loss rate, error rate, stability, cost and distance values for each link. The flow-specific information comprises source address, session ID, destination address and QoS requirements of the flow. Because of volatile behaviour in network topology and channel characteristics, these state information are intrinsically imprecise. This may cause inaccurate routing decisions resulting in some packets missing their deadlines, leading to violation of real-time QoS commitment [22].

Differentiation in QoS services: QoS requirement varies with service requirement. Hence to develop a single holistic approach for enabling QoS effectively is very challenging.

\section{QUEUEING APPROACH}

Queuing approach is the process of handling the sequence of activities (Packets) that arrives to certain router (mobile node) in certain shape. It defines the order in which they are served and the way in which resources are divided among packets. To transmit a packet, the router must implement some queueing mechanism which controls the whole transmission course of action. These mechanisms affect the packets latency experienced by determining how long a packet must waits before its transmission. Examples of the some common queuing mechanism are the first in first out (FIFO) which is called Drop Tail (DT) in networking, The shortest is served first (SJF), last in first out (LIFO), priority queuing (PQ), round robin, service in random order, random exponential marking (REM) and others [23]. Queuing models are constructed to help the researchers, scientists and the engineers to analyze the performance of complex dynamic systems. The purpose of building a queuing 
model is to obtain reliable statistics that can be used to analyze, evaluate, improve the performance and examine the behavior of any complex wireless networks like MANET [24]. Therefore, many performance metrics were developed to collect and report the required information to measure the performance of the MANETs. All of the measuring performance processes requires the use of statistical modelling to determine the results. This study deals with the discussion of important performance metrics in MANET for QoS provision.

Throughput: In MANETs throughput is measure as a rate of successful packets delivery over a wireless channel. This data can be forwarded over logical link, or pass through a certain network node. The throughput is generally measured in bits per second (bps or bit/s), or sometimes in data packets per second, or data packets per time slot.

Throughput= Total packet received/ amount of forwarded packet over certain time interval

Dropped Packets: These are the number of packets that sent from the source node and fail to reach the destination node [25].

Dropped packets $=$ sent packets from source - received packets at destination

Mean inter arrival time: Mean inter-arrival time (av) is the summation of inter-arrival times by the number of received packets. If $\boldsymbol{Y}$ is arrival time of the packet and $\mathrm{n}$ is the number of received packets.

\section{$a v=\sum \frac{\gamma}{n}$}

Average end to end delay: end to end delay (ED) represents the time required to move a packet from source node to destination node. The average end to end delay can be calculated by summing the times taken by all received packets divided by its total numbers [25]. It is the accumulation of queuing delay, transmission delay and end system processing delay in mobile node.

\section{$E D=\sum$ (received time-sent time)/ $\sum$ (number of packets)}

Latency: amount of time experienced by a packet from source to reach the destination.

Jitter: In MANET, packet jitter is measured as an average of the variation in latency from the network mean latency. However, the standard based term is packet delay variation (PDV), which is an important QoS factor in assessment of network performance. A network with constant latency has no jitter. Here Di+1 is the delay of $\mathrm{ith}+1$ packet and Di is the delay of ith packet.

$$
P D V=D i+1-D i
$$

Packet Delivery Fraction (PDF): It can be expressed as the ratio of the delivered packets at destination to the packets sent from the source node [Imran Khan et. al. 2006].

$$
P D F=100 * \text { (Number of received packets / Number of sent packets) }
$$

Scalability: Typically in traditional network architecture, even with perfect network state information, queueing delay makes the network and application decision suboptimal which sometime leads to network collapse. Although, the heterogeneous networks consist of different nodes with different resource but still has better scalability as compared to homogenous networks. Homogenous networks even, are easy to model and analyze but they exhibits outdated and sub optimal decision which leads to poor scalability as shown in the following Figure 2.

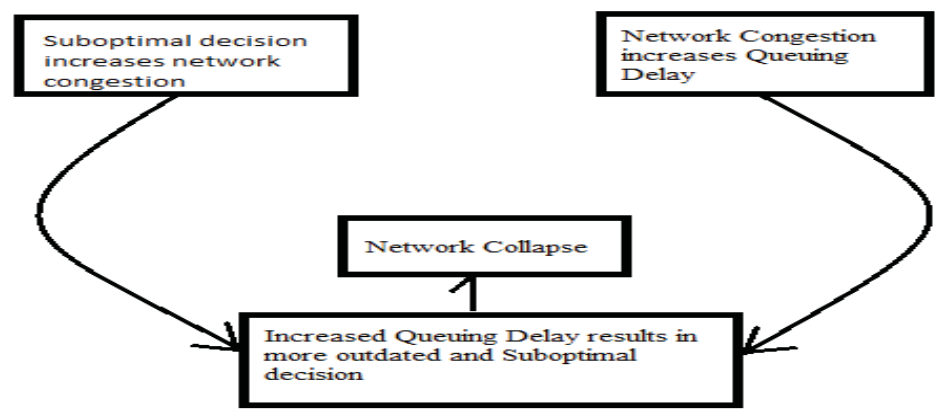

Figure 2: General view MANET scalability

\section{CONCLUSION}

In this paper, we have discussed various issues and challenges for MANETs. We have also reviewed different queueing models for MANETs and presented various QoS metrics based on the available models. 


\section{REFERENCES}

[1] T. Larsson, Routing Protocols in Wireless Ad hoc Networks: A Simulation Study, Master's thesis, Lulea University of Technology, Sweden, 1998.

[2] Chhagan Lal, V. Laxmi, M.S. Gaur, Building Next Generation Converged Networks: Theory and Practice, Pathan (Ed.), Taxonomy of QOS Aware Routing Protocols for MANETs, (NY, CRC Press, 2013), 533-560

[3] Bhatnagar J. et. al., Delay Aware Routing Solutions for MANETs: A Survey, International Journal of Advanced Research in Computer Science and Software Engineering, 3(7), July, 2013, ), 1021-1025.

[4] Baskar, S, S. Palaniammal, Queueing Models in MANETs, American Journal of Applied Sciences $11 \quad$ (2), 2014, 308-315.

[5] Seema Kumari, M. U Kharat, Delay analysis of multihop wireless ad hoc network using queuing network model, International Journal of Computers and Technology, 10(4), Aug 15, 2013, 1503-1509.

[6] Enass Fadil, Saad Talib Hasson, Queueing approach to Model the MANETs performance, 6 (2), Sep 2012, 18-24.

[7] Vishal Jain, R.B. Lenin and Sanjay Srivastava, Modelling MANETs using queueing networks, In Proc. of NCC 2007, IIT Kanpur, January 2007.

[8] Gaurav Khandelwal, Giridhar Prasanna, Chittaranjan Hota, Probabilistic Routing Using Queueing Theory For MANETs, International Journal of Wireless and Mobile Networks, 3(4), 2011, 144-158.

[9] Ahyoung Lee, Ilkyeun Ra, A Queueing Network Model Based on Ad hoc Routing Networks for Multimedia Communications, Applied Mathematics \& Information Sciences, 6(1), 2012. 271-283.

[10] N. Bisnik. Alhussein Abouzied, Queuing Delay and Achievable Throughput in Random Access Wireless Ad Hoc Networks, IEEE In proc. International Conference of Wireless Communications and Mobile Computing, Vancouver, Canada, 2006, 773-778.

[11] R. Ell-Khoury, Richard El- Azouzi, Eitan Altman, Delay analysis for real-time streaming media in multi-hop ad hoc networks, IEEE , In proc. International Symposium,on Modeling and Optimization in Mobile Ad hoc Network, Wireless Network (WiOPT), Germany, 2008.

[12] Kamal K. Sharma et. al., Modeling and Analysis of End-To-End Delay for Ad Hoc Pervasive Multimedia Network, Proc. of International Multi Conference of Engineers and Computer Scientists, IMECS 2010, Hong Kong, March 17-19.

[13] M. Ozdemir, B. McDonald, An M/MMGI/1/K queuing model for IEEE 802.11 ad hoc networks, in Proceedings of the 1st ACM International Workshop on Performance Evaluation of Wireless Ad Hoc, Sensor, and Ubiquitous Networks, page,. ACM Press, 2004, $107-111$.

[14] A. K. Dey, D. Salber, G. D. Abowd, A conceptual framework and a toolkit for supporting the rapid prototyping of context-aware applications, Human-Computer Interaction, 16(2-4), 2001, 97-166.

[15] J. Li, C. Blake, D. S. D. Couto, H. I. Lee, and R. Morris, Capacity of ad hoc wireless networks, Proceedings of the 7th annual international conference on Mobile computing and networking (MobiCom 2001), ACM Press, New York, NY, USA, 2001, 61-69.

[16] Yuanfeng W. et. al., A Queueing Theory Based Approach to QoS-Driven Adaptation for Service Discovery over MANETs, IEEE 15th International Conference on Computational Science and Engineering, 2012, 594-601.

[17] M. Barbeau, E. Kranakis, and H. Luo, Strategies for service discovery over ad hoc networks, Engineering Letters, 13, 2006.

[18] D. Chakraborty, A. Shenoi, Y. Yesha, Y. Yesha, A. Joshi, A queueing theoretic model for service discovery in ad-hoc networks, Communication Networks and Distributed Systems, Modeling and Simulation Conference (CNDS), January 2004, 1-7.

[19] Vidhyasanker, B.S. Manoj, Siva Ram Murthy, Slot Allocation Schemes for Delay-Sensitive Traffic Support in Asynchronous Wireless Mesh Networks, International Journal of Computer and Telecommunications Networking, 50(15), October 2006, 2595-2613.

[20] Z. Wang, J. Crowcroft, Quality-of-service routing for supporting multimedia applications, IEEE journal on Selected Areas in Communications, 14(7), 1996, 1228- 1234.

[21] C Sreedhar, Varun Verma Sangaraju, A Survey On Security Issues In Routing In MANETS, International Journal of Computer \& Organization Trends, 3(9), October 2013, 399-403.

[22] Yuanfeng W. et. al., A Queueing Theory Based Approach to QoS-Driven Adaptation for Service Discovery over MANETs, IEEE 15th International Conference on Computational Science and Engineering, 2012, 594-601.

[23] S. Athuraliya, Victor H. Li, Steven H. Low, Qinghe Yin, REM: Active Queue Management, IEEE Network, Australia, 2001, 1-12.

[24] B. Filipowicz, J. Kwiecien, Queueing systems and networks. Models and applications, Bulletin of the Polish Academy of Technical Sciences, 56(4), Poland, 2008, 379-390.

[25] Aliff Umair Salleh, Zulkifli Ishak, Norashidah Md. Din, Md Zaini Jamaudin, Trace Analyzer for NS-2, SCOReD IEEE, Malaysia, 2006, 29-32. 